\title{
Identification of novel mutations in endometrial cancer patients by whole-exome sequencing
}

\author{
YA-SIAN CHANG ${ }^{1-3}$, HSIEN-DA HUANG ${ }^{4,5}$, KUN-TU YEH $^{6}$ and JAN-GOWTH CHANG ${ }^{1,2,7}$ \\ ${ }^{1}$ Epigenome Research Center; ${ }^{2}$ Department of Laboratory Medicine, China Medical University Hospital, Taichung 404; \\ ${ }^{3}$ Department of Medical Laboratory Science and Biotechnology, China Medical University, Taichung 404; \\ ${ }^{4}$ Department of Biological Science and Technology; ${ }^{5}$ Institute of Bioinformatics and Systems Biology, \\ National Chiao Tung University, Hsinchu 300; ${ }^{6}$ Department of Pathology, Changhua Christian Hospital, \\ Changhua 500; ${ }^{7}$ School of Medicine, China Medical University, Taichung 404, Taiwan, R.O.C.
}

Received December 2, 2016; Accepted March 7, 2017

DOI: 10.3892/ijo.2017.3919

\begin{abstract}
The aim of the present study was to identify genomic alterations in Taiwanese endometrial cancer patients. This information is vitally important in Taiwan, where endometrial cancer is the second most common gynecological cancer. We performed whole-exome sequencing on DNA from 14 tumor tissue samples from Taiwanese endometrial cancer patients. We used the Genome Analysis Tool kit software package for data analysis, and the dbSNP, Catalogue of Somatic Mutations in Cancer (COSMIC) and The Cancer Genome Atlas (TCGA) databases for comparisons. Variants were validated via Sanger sequencing. We identified 143 non-synonymous mutations in 756 canonical cancer-related genes and 1,271 non-synonymous mutations in non-canonical cancer-related genes in 14 endometrial samples. PTEN, KRAS and PIK3RI were the most frequently mutated canonical cancer-related genes. Our results revealed nine potential driver genes (MAPT, IL24, MCM6, TSC1, BIRC2, CIITA, DST, CASP8 and NOTCH2) and 21 potential passenger genes (ARMCX4, IGSF10, VPS13C, DCT, DNAH14, TLN1, ZNF605, ZSCAN29, MOCOS, CMYA5, PCDH17, UGT1A8, CYFIP2, MACF1, NUDT5, JAKMIP1, PCDHGB4, FAM178A, SNX6, IMP4 and PCMTD1). The detected molecular aberrations led to putative activation of the mTOR, Wnt, MAPK, VEGF and ErbB pathways, as well as aberrant DNA repair, cell cycle control and apoptosis pathways. We characterized the mutational landscape and genetic alterations in multiple cellular pathways of endometrial cancer in the Taiwanese population.
\end{abstract}

Correspondence to: Dr Jan-Gowth Chang, Epigenome Research Center, China Medical University Hospital, 2 Yuh-Der Road, Taichung 404, Taiwan, R.O.C.

E-mail: d6781@mail.cmuh.org.tw

Key words: whole-exome sequencing, endometrial cancer, driver genes, passenger genes

\section{Introduction}

Endometrial cancer is the most common malignancy of the female genital system in developed countries. In Taiwan, endometrial cancer ranks second among gynecological cancers in its incidence. Risk factors for endometrial cancer are both environmental and genetic. One major environmental risk factor is endogenous or exogenous estrogen exposure: many sources of exogenous estrogen exist, including oral contraceptives and hormone replacement therapy (1). Some genetic variants involved in the steroid hormone biosynthesis and metabolism pathways may contribute to hyperestrogenic status, which is associated with endometrial cancer risk $(2,3)$. Use of oral contraceptives provides significant long-term protection against endometrial cancer (4). Long-term sequential estrogen-plus-progestin therapy during menopause increases the risk of endometrial cancer, whereas short-term estrogen-plus-progestin use in menopause decreases the risk of endometrial cancer (5). Endometrial cancer patients who received extended adjuvant tamoxifen do not appear to be at greater risk for endometrial cancer (6).

Next-generation sequencing (NGS) will play an important role in anticancer drug development (7) and targeted therapy (8). At present, there are multiple NGS instruments in use, such as the Illumina HiSeq and MiSeq (Illumina, Inc., San Diego, CA, USA), the Ion Torrent Proton and Personal Genome Machine (Life Technologies, Carlsbad, CA, USA), as well as the Roche 454 Sequencer and GS Junior (Roche Applied Biosystems, Nutley, NJ, USA). The Cancer Genome Atlas (TCGA) project used NGS techniques to identify hundreds of somatically altered genes (9-12). This technical advance is rapidly altering the routine practice of molecular pathology, from single-gene tests (i.e., Sanger sequencing to assess KRAS mutations in colorectal cancer) to multiplexed NGS assays. Several NGS approaches have been commonly implemented clinically in oncology, including hybrid capture-based panels, multiplexed polymerase chain reaction (PCR)-based panels and comprehensive genome/ transcriptome/exome sequencing (13-16). Whole genome sequencing offers high throughput, high accuracy $(<1$ error per $100 \mathrm{~kb})$ and affordable cost $(<\$ 5,000$ USD in reagents) $(17)$. Recently, the American College of Medical Genetics and 
Table I. Summary of sequencing alignment and coverage statistics in the exomes of 14 endometrial tumors.

\begin{tabular}{lccccccc}
\hline $\begin{array}{l}\text { Patient } \\
\text { ID }\end{array}$ & $\begin{array}{c}\text { Total } \\
\text { raw reads }\end{array}$ & $\begin{array}{c}\text { Total } \\
\text { effective reads }\end{array}$ & $\begin{array}{c}\text { Reads mapped } \\
\text { to genome }\end{array}$ & $\begin{array}{c}\text { Total effective } \\
\text { yield }(\mathrm{Mb})\end{array}$ & $\begin{array}{c}\text { Average read } \\
\text { length (bp) }\end{array}$ & $\begin{array}{c}\text { Covered } \\
\geq 20 \mathrm{X}(\%)\end{array}$ & $\begin{array}{c}\text { Average sequencing } \\
\text { depth on target }\end{array}$ \\
\hline F105T & $20,976,408$ & $14,817,961$ & $14,725,603$ & 45974.96 & 200.01 & 43.06 & 19.43 \\
F139T & $18,071,410$ & $12,999,809$ & $12,909,672$ & 43438.89 & 200.01 & 51.07 & 21.78 \\
F141T & $18,308,457$ & $13,180,222$ & $13,077,252$ & 45838.97 & 200.10 & 44.58 & 19.06 \\
F61 & $21,965,842$ & $15,337,823$ & $15,247,340$ & 47366.56 & 200.00 & 45.76 & 20.01 \\
F114T & $18,690,475$ & $13,546,119$ & $13,433,300$ & 46105.01 & 200.16 & 48.37 & 20.01 \\
F123 & $20,115,993$ & $14,018,792$ & $13,919,598$ & 46067.53 & 199.99 & 43.44 & 18.83 \\
F132 & $21,510,383$ & $14,710,924$ & $14,621,813$ & 45507.75 & 199.83 & 45.5 & 20.56 \\
F134 & $20,126,153$ & $14,005,289$ & $13,909,284$ & 45658.48 & 199.98 & 42.77 & 18.94 \\
F146 & $26,158,898$ & $18,289,158$ & $18,193,144$ & 50310.04 & 200.03 & 46.31 & 21.53 \\
F147T & $19,394,632$ & $14,099,623$ & $13,962,761$ & 45656.60 & 200.17 & 48.56 & 20.92 \\
F150T & $20,623,512$ & $14,156,494$ & $14,080,096$ & 45931.50 & 199.85 & 48.22 & 20.58 \\
F152T & $19,092,410$ & $14,153,725$ & $14,048,858$ & 46229.92 & 200.28 & 50.62 & 21.11 \\
F92T & $18,726,658$ & $13,243,029$ & $13,164,997$ & 45153.23 & 200.09 & 43.91 & 18.60 \\
03-3812T & $17,550,923$ & $12,789,944$ & $12,748,550$ & 44627.55 & 200.18 & 52.99 & 21.17 \\
Average & $20,093,725$ & $14,239,208$ & $14,145,876$ & 45990.50 & 200 & 47.03 & 20 \\
\hline
\end{tabular}

Genomics (ACMG) recommended reporting pathogenic findings in 56 genes with low coverage. Low sequencing coverage may contribute to false negative clinical exome results (18).

Based on histological subtype, endometrial cancer is classified into two major types (I and II). Type I (endometrioid) carcinoma is the most frequent type of endometrial cancer, accounting for over $80 \%$ of cases. Type II (non-endometrioid) carcinoma comprises a minority of endometrial cancer cases. Whole-exome sequencing (WES) has led to the discovery of ARIDIA as a novel regulator of PI3K pathway activity in endometrioid endometrial cancer (19). Through an integrated genomic, transcriptomic and proteomic analysis of endometrial cancer, TCGA research has suggested a novel molecular classification for two histological subtypes (12).

We performed WES on 14 patients with endometrial cancers. Our results identify cancer driver and passenger genes, and adhere to ACMG recommendations for the examination and reporting of secondary genetic findings during clinical genomic testing.

\section{Materials and methods}

Patients and samples. Fourteen patients with endometrial cancer were recruited for this study. DNA was extracted using the QIAamp DNA Micro kit (Qiagen, Heidelberg, Germany) following the manufacturer's protocol. Extracted DNA samples were quantified using NanoDrop 2000 spectrophotometer (Thermal Fisher Scientific, Waltham, MA, USA) and a Qubit fluorometer (Invitrogen, Carlsbad, CA, USA). This study was approved by the Institutional Review Board of Kaohsiung Medical University Hospital (KMUH-IRB-970488).

Whole-exome sequencing. To generate standard exome capture libraries, we used the Agilent SureSelect XT Reagent kit protocol for the Illumina Hiseq paired-end sequencing library (cat. no. G9611A). In all cases, the SureSelect XT Human All Exon Version $4(51 \mathrm{Mb})$ probe set was used. We used $50 \mathrm{ng}$ genomic DNA for library construction with the Agilent SureSelect XT Reagent kit. The adapter-ligated sample was purified using Agencourt AMPure XP beads (Beckman Coulter, Brea, CA, USA) and analyzed on a Bioanalyzer DNA 1000 chip. Part of the sample (750 ng) was prepared for hybridization with the capture baits, and the sample was hybridized for $90 \mathrm{~min}$ at $65^{\circ} \mathrm{C}$, captured with the Dynabeads MyOne Streptavidin T1 (Life Technologies) and purified using Agencourt AMPure XP beads. The Agilent protocol was used to add index tags by post-hybridization amplification. Finally, all samples were sequenced on an Illumina Hiseq system using 100 PE protocol. Metadata were deposited in the NCBI Sequence Read Archive under the accession no. SRP099176.

Data analysis. To filter low-quality reads, we used the FASTXToolkit (http://hannonlab.cshl.edu/fastx_toolkit) to process the raw read data files. There were two steps of sequence quality processing. The command 'fastq_quality_filter -Q33 -q 30 -p 70'. '-q 30' indicated that the minimum quality score was 30. '-p 70' meant that bases must have a '-q' quality score of $70 \%$ or greater. Sequences were retained if both forward and reverse sequencing reads passed the first step.

An efficient sequence alignment tool, Bowtie2, was used to align the retained reads with human genome (Grch38.p2) (20). Based on the results of sequence alignment, reads having only one chromosome location were retained for further analysis. The Genome Analysis Tool kit, is a widely used discovery tool to identify genetic variants based on the results of sequence alignment (21).

Several databases and tools were used to annotate identified genetic variants. dbSNP (b144) is an archive of genetic variation within different species that provides information about each genetic variant (22). ClinVar is a database of clinically significant genetic variants (23). COSMIC (v73) collects somatic mutation information for human cancers (24). The TCGA project collects genomics, methylomic and transcriptomic data across cancer types (25). Integrated mutation 
prediction software (PolyPhen-2 and SIFT) were used for analyses of the identified variants $(26,27)$. DrGaP was used to identify driver genes and driver signaling pathways (28).

Confirmation by Sanger sequencing. Potential mutations identified by whole-exome sequencing were confirmed by PCR and Sanger sequencing. Specific PCR primers were designed using Primer3 software (primers can be provided on request). The products were sequenced directly with the ABI PRISM terminator cycle sequencing kit v3.1 on an ABI 3130 DNA sequencer (Applied Biosystems, Carlsbad, CA, USA).

\section{Results}

Whole-exome sequence analysis and coverage. Using massive parallel sequencing on a HiSeq platform, we generated $\sim 45$ billion bases of effective sequence data with an average read length of 200 bases. After mapping to the human reference genome (Grch38.p2) using the Bowtie 2 alignment tool, we obtained an average depth of coverage for the target regions of 20x for each sample (Table I). The false positive and false negative rates were estimated to be $2.75 \%$ (12/437) and $19.69 \%$ (76/386), respectively, after confirmed by target sequencing of cancer-related genes (29). Table II provides an overview of our approach to identify variants.

Mutational landscape in the 756 canonical cancer-related genes. The 143 non-synonymous mutations identified in the present study occurred in 129 genes, and included 141 missense mutations, one nonsense mutation and one frame shift mutation (details can be provided on request). The most frequently mutated genes were PTEN (35.71\%; 5/14), KRAS and PIK3RI (14.29\%, 2/14).

Sixty-eight variants already existed in the dbSNP, COSMIC, or TCGA databases and 75 variants in 71 genes did not. In addition, we found 13 different mutations in the same codon of 12 canonical cancer-related genes compared with the TCGA database (CDK8, DPYD, EPHA3, EPHA6, FLNA, MAPK1, MAPK7, PLK1, PTEN, RAPGEF2, RFC4 and ZNF521) (details can be provided on request).

We ordered the 75 new variants according to sequencing depth. Next, we selected 12 variants for Sanger sequencing. Six variants had relatively high depth (208x, 156x, 131x, 105x, 97x and $89 x)$. Several novel mutations were present only in cancer tissue, including MAPT p.C432Y, IL24 p.G192W, MCM6 p.R263H, TSC1 p.D1054G and BIRC2 p.P152S. However, $K A T 6 B$ p.S1693N was also detected in paired non-cancerous tissues. Six variants had low depth (22x, 21x, 21x, 20x, 20x and 20x). Novel mutations present only in cancer tissue including CIITA p.E1063D, DST p.Q2014H, CASP8 p.L219M and NOTCH2 p.M2054I. FLNA p.V528L and HSPAIL p.P93H were also detected in paired non-cancerous tissues (Fig. 1).

Mutational landscape in the non-canonical cancer-related genes. The 1,271 non-synonymous mutations identified in this study, including 1,270 missense mutations and one nonsense mutation, were located at 1,144 genes. In total, 723 variants had previously been reported in the dbSNP, COSMIC or TCGA databases, while 548 variants in 516 genes had not (details can be provided on request). 
A

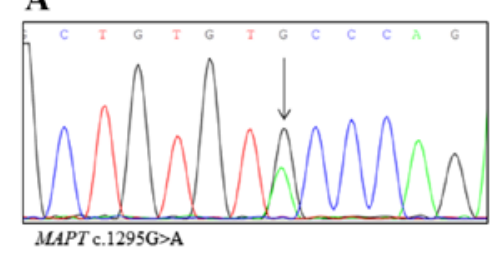

D

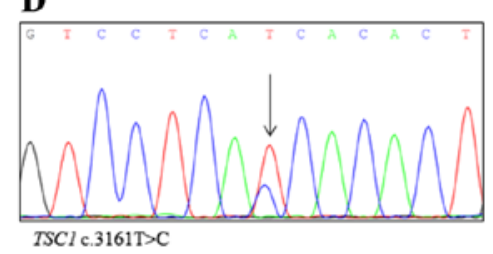

G

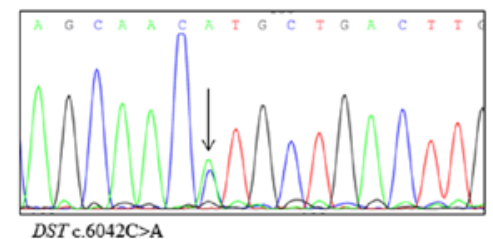

B

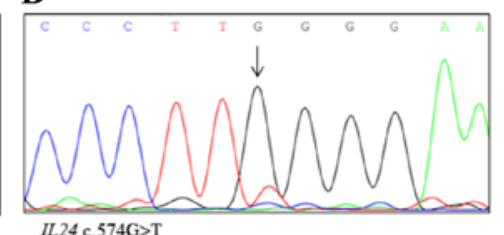

IL 24 c.574G $>$ T

E

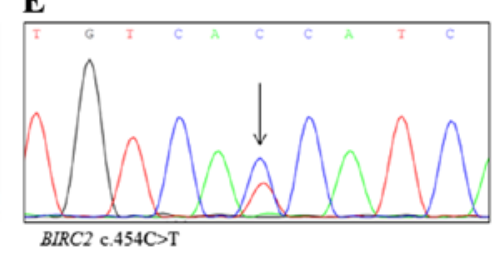

H

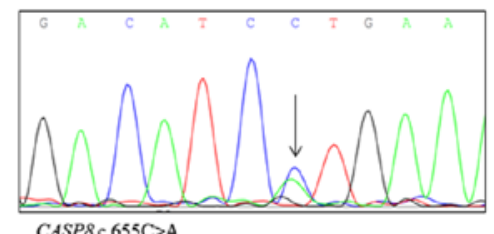

C

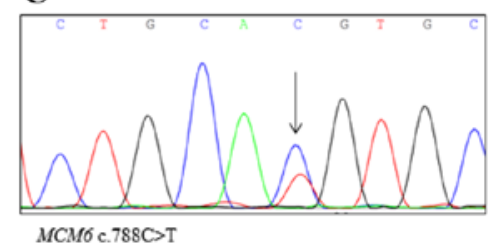

F

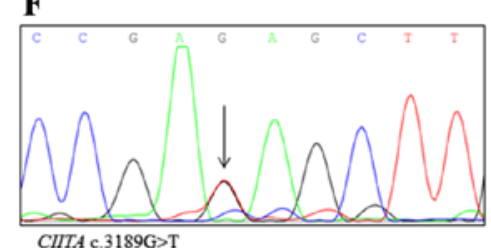

I

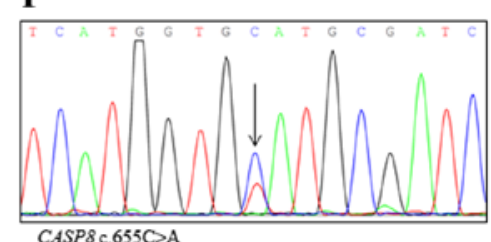

Figure 1. Confirmatory analysis by Sanger sequencing of canonical cancer related genes detected via WES. (A) MAPT, (B) IL24, (C) MCM6, (D) TSC1, (E) BIRC2, (F) CIITA, (G) DST, (H) CASP8 and (I) NOTCH2.

However, nearly all mutations had been reported in the 542 TCGA endometrial cancer samples. Only three mutations had never been reported (ARMCX4, CUTA and SAP3OL). We selected these mutations for Sanger sequencing. CUTA p.T187R and SAP3OL p.H77Y were also detected in paired non-cancerous tissues; $A R M C X 4$ p.P2056H was the sole novel mutation that was detected only in cancer tissue (Fig. 2). We also found 49 different mutations in the same codon of 49 non-canonical cancer-related genes compared with the TCGA database (details can be provided on request).

The 1,144 genes were divided into five groups according to mutation frequency. Fifteen non-canonical cancer-related genes had high frequencies of mutation $(35.71-50 \% ; 5-7 / 14)$ and four variants in three genes (IGSF10, FBXL13 and PRUNE2) were novel. We selected these new genetic variants for Sanger sequencing. We detected IGSF10 p.R455W, FBXL13 p.G313A and PRUNE2 p.S2439I in paired non-cancerous tissues, while IGSF10 p.K188Q was detected only in cancer tissue (Fig. 2).

Six genes were mutated in $4(28.57 \%)$ of the 14 patients. One gene $(V P S 13 C)$ had a new genetic variant. Sanger sequencing confirmed that VPS13C p.V2017M was a novel mutation detected only in cancer tissue (Fig. 2).

Twenty-two genes were mutated in $3(21.43 \%)$ of the 14 patients, and there were 12 new genetic variants in 8 genes. We carried out Sanger sequencing on the new variants, and found that ARAP2 p.Y1491C, DNAH14 p.D4225V, TLN1 p.D1325E, TREH p.M196T and ZSCAN29 p.G304V were also present in paired non-cancerous tissues. Sanger sequencing also confirmed that $D C T$ p.Y548S, DNAH14 p.G1566C, TLN1 p.A555V, ZNF605 p.P283Q, p.E652A and ZSCAN29 p.P71S were novel mutations present only in cancer tissue (Fig. 2). One variant (TRANK1 p.W2445L) had an incorrect base call.

One hundred and nine genes were mutated in $2(14.29 \%)$ of the 14 patients, and there were 73 new genetic variants in 54 genes. We ordered the 73 new variants according to sequencing depth. Next, we selected 10 variants for Sanger sequencing. Five variants had high depth (163x, 108x, 104x, 99x and 92x). Several novel mutations were detected only in cancer tissue, including MOCOS p.I320T, CMYA5 p.P2149H and PCDH17 p.P976H (Fig. 2). MAGEE2 p.F249L and CRYBG3 p.Q632E were also detected in paired non-cancerous tissues. Five variants had low depth (all 20x). Novel mutations present only in cancer tissue including UGT1A8 p.Y290C and CYFIP2 p.C959Y (Fig. 2), while APOL4 p.A316V and TRIM26 p.Q197H were also detected in paired non-cancerous tissues. One variant (MYO3B p.G230W) had an incorrect base call.

Among low-frequency mutations $(7.14 \% ; 1 / 14)$ in 992 noncanonical cancer-related genes, there were 458 new genetic variants in 450 genes. We ordered these 458 variants according to sequencing depth. Next, we selected 10 variants for Sanger sequencing. Five variants had high depth (286x, 244x, 223x, $197 x$ and 187x). Several novel mutations were detected only in cancer tissue, including MACF1 p.Q181H, NUDT5 p.G135S, JAKMIPI p.R352M and PCDHGB4 p.L154I (Fig. 2). MYH10 p.L1048X was also detected in paired non-cancerous tissues. Five variants had low depth (all 20x). Novel mutations detected only in cancer tissue included FAM178A p.T86I, SNX6 p.L38R, IMP4 p.T163M and PCMTD1 p.A41D (Fig. 2). TNFAIP8L3 p.A38T was also detected in the paired non-cancerous tissues.

ACMG gene. Of the 14 endometrial cancer samples, $28.57 \%$ (4/14) harbored ACMG mutations, including RYR2, MSH6 and TSCl, but did not have mutations in the remaining 53 genes. Mutations in the RYR2 gene were detected in $14.29 \%$ $(2 / 14)$ of endometrial cancer specimens, with mutations at p.G1209R (rs770286824) and p.F4960L (a novel mutation) identified in our endometrial cancer samples. Both the MSH6 mutation at p.Q572H (rs745772518) and the TSC1 mutation 
A

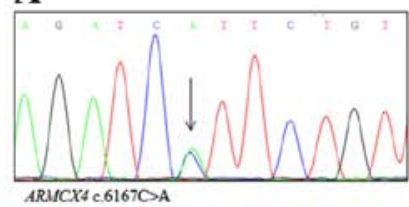

E

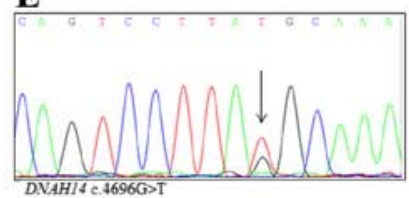

H

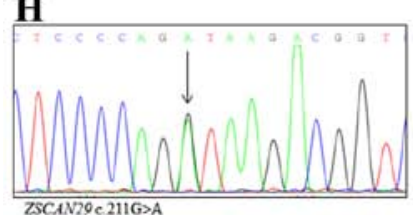

L

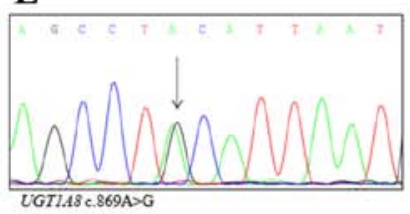

$\mathbf{P}$

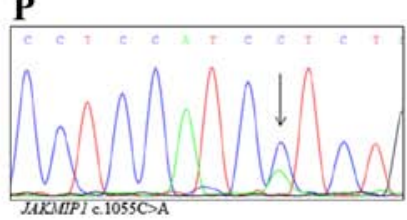

T

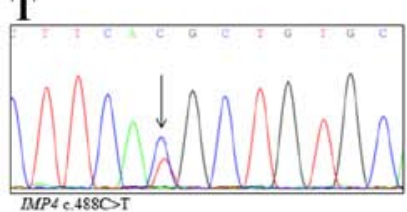

B

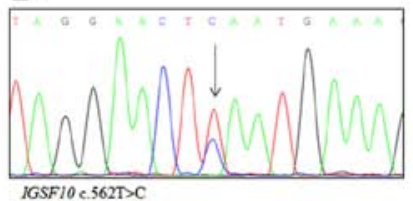

$\mathbf{F}$
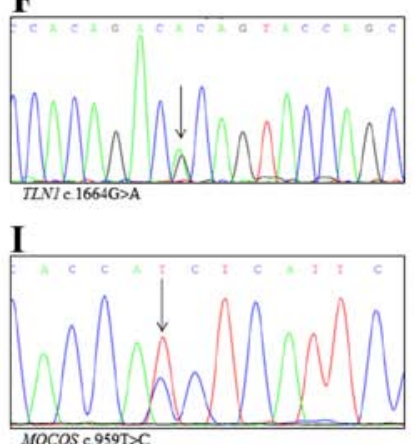

M

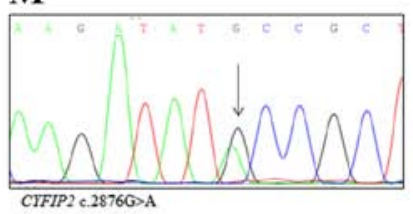

$\mathbf{O}$

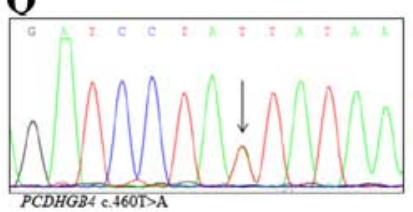

$\mathbf{U}$

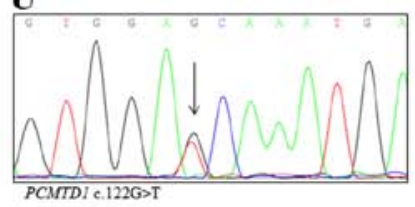

C

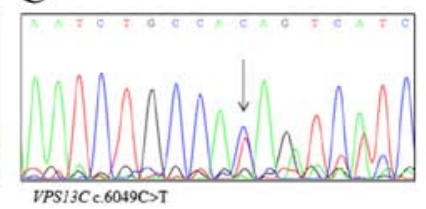

G

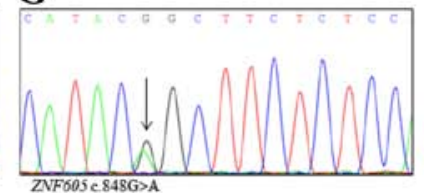

J

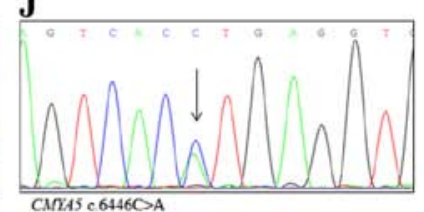

$\mathbf{N}$

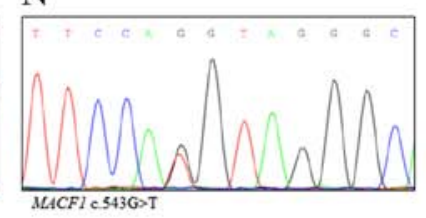

R

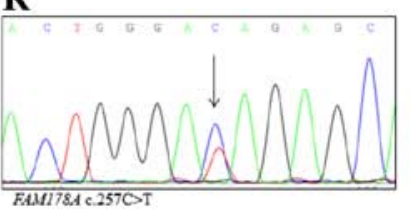

D

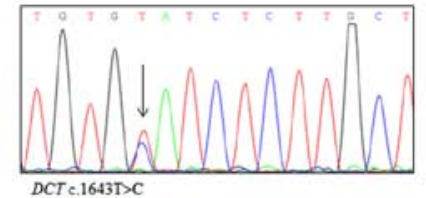

DCT C.1643T>C

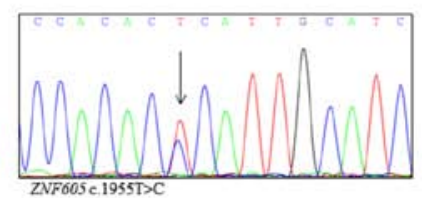

K

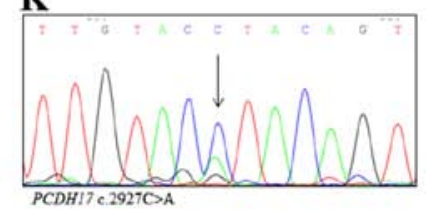

O

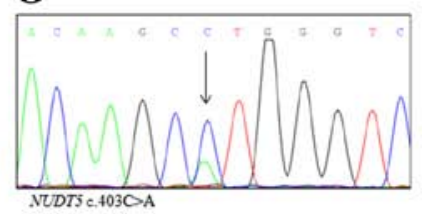

S

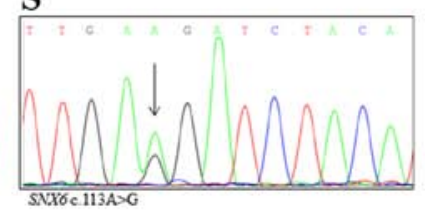

Figure 2. Confirmatory analysis by Sanger sequencing of non-canonical cancer related genes detected via WES. (A) ARMCX4, (B) IGSF10, (C) VPS13C, (D) DCT, (E) DNAH14, (F) TLN1, (G) ZNF605, (H) ZSCAN29, (I) MOCOS, (J) CMYA5, (K) PCDH17, (L) UGT1A8, (M) CYFIP2, (N) MACF1, (O) NUDT5, (P) JAKMIP1, (Q) PCDHGB4, (R) FAM178A, (S) SNX6, (T) IMP4 and (U) PCMTD1.

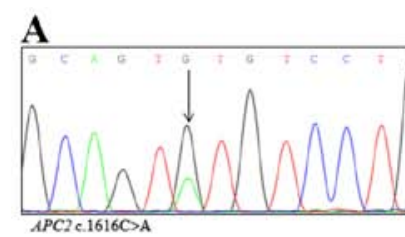

E

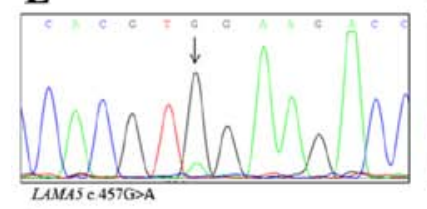

I

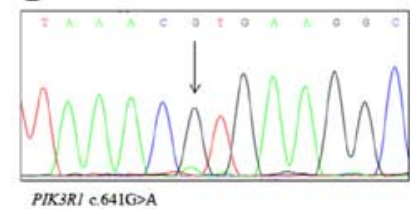

B

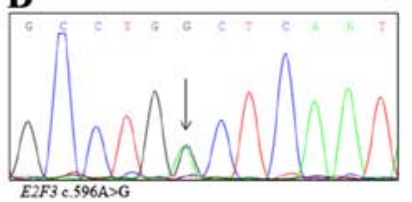

F

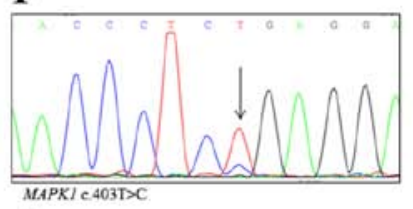

J

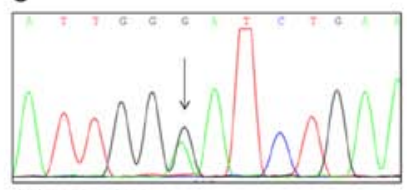

C

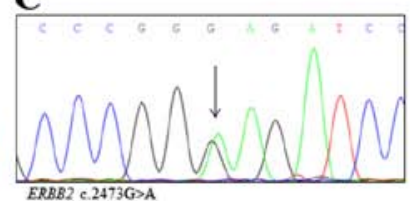

G

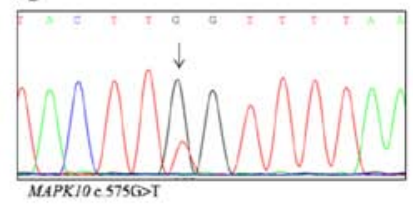

D

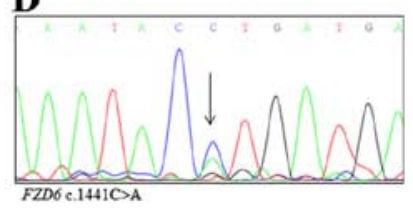

H

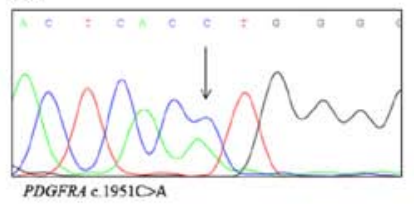

Figure 3. Confirmatory analysis by Sanger sequencing of KEGG cancer pathway (hsa05200) detected via WES. (A) APC2, (B) E2F3, (C) ERBB2, (D) FZD6, (E) LAMA5, (F) MAPK1, (G) MAPK10, (H) PDGFRA, (I) PIK3R1 and (J) SOS2. 


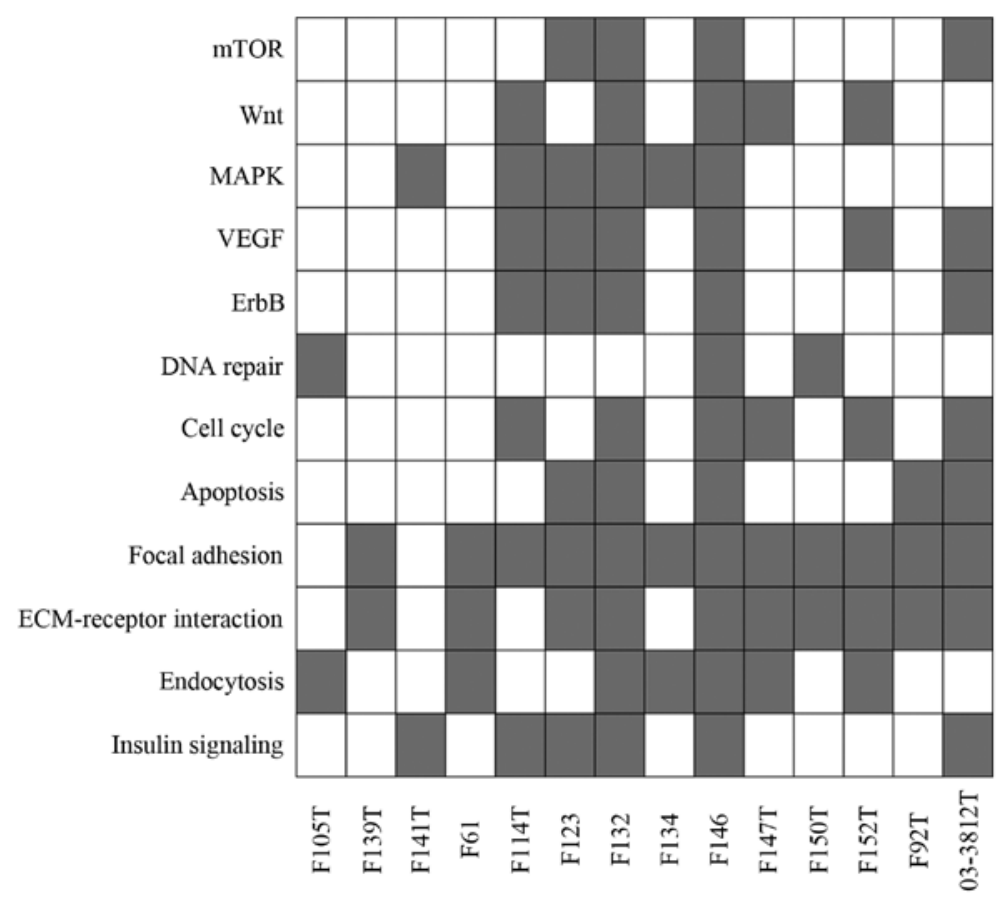

Figure 4. Altered pathways for 14 Taiwanese patient samples. The definition of altered pathway is at least 1 altered gene in the pathway.

at p.D1054G were detected in $7.14 \%(1 / 14)$ of endometrial cancer samples.

Altered pathways. Functional annotation of the 1,273 mutated genes was performed using the DrGaP tool. Thirty-seven of the 1,273 mutated genes were found in the Kyoto Encyclopedia of Genes and Genomics (KEGG) cancer pathways (hsa05200), including APC2, BCR, BIRC2, CASP8, CCNA1, CREBBP, CTNNA2, CTNNA3, CTNNB1, E2F3, ERBB2, FGFR2, FOXO1, FZD6, HHIP, ITGA2, ITGA3, KRAS, LAMA1, LAMA2, LAMA3, LAMA5, LAMC1, LAMC2, MAPK1, MAPK10, MSH6, MTOR, PDGFRA, PIK3CA, PIK3R1, PLCG2, PTEN, SOS2, STAT5A, TCF7 and TRAF5. We used Sanger sequencing validation to confirm new variants (Fig. 3). Moreover, we identified several cellular pathways that were altered in endometrial cancer tissues and we found that each sample had at least two pathways involved in the carcinogenesis of endometrial cancer (Fig. 4).

\section{Discussion}

The present study described somatic mutation in the whole endometrial cancer exome. We identified several cancer driver and passengers genes from canonical and non-canonical cancer-related genes. Overall, 35.71\% of endometrial cancer cases harbored PTEN mutations. Mutations were also found in other canonical cancer-related genes, including KRAS and PIK3Rl (14.29\% each). To the best of our knowledge, several sequencing variants have not been reported, including canonical cancer-related genes (MAPT, IL24, MCM6, TSC1, BIRC2, CIITA, DST, CASP8 and NOTCH2) and non-canonical cancerrelated genes (ARMCX4, IGSF10, VPS13C, DCT, DNAH14, TLN1, ZNF605, ZSCAN29, MOCOS, CMYA5, PCDH17, UGT1A8, CYFIP2, MACF1, NUDT5, JAKMIP1, PCDHGB4, FAM178A, SNX6, IMP4 and PCMTD1).
Two canonical cancer-related genes (TSC1 and BIRC2) were found in the Oncomine Cancer Research Panel, which was used in the National Cancer Institute Match Trial (30). TSC1 is a tumor suppressor gene that encodes a growth inhibitory protein (hamartin) thought to play a role in the stabilization of tuberin. TSCl is a gene involved in the mTOR pathway. Mutations in TSC1, TSC 2 and MTOR have been associated with response to rapalogs in patients with metastatic renal cell carcinoma (31). We identified a novel mutation, p.D1054G, in one endometrial cancer patient.

$B I R C 2$ is an oncogene that encodes c-IAP1, which is a member of the apoptosis inhibitor family. Members of this family inhibit apoptosis by binding to TRAF1 and TRAF2 and likely interfering with activation of ICE-like proteases. Previously, Choschzick et al (32) reported BIRC2 amplification in uterine cervix cancer. In the present study, we identified a novel mutation, p.P152S, in a patient with endometrial cancer.

The genetic alterations we found involved the mTOR, Wnt, MAPK, VEGF and ErbB pathways, as well as aberrant DNA repair, cell cycle control and apoptosis pathways. These pathways have previously been shown to be involved in the multistep development of endometrial cancer, and clinical trials of drugs for endometrial cancer that target these pathways have been carried out (33). We also found genetic alterations in the steroid hormone biosynthesis pathway (hsa00140), including AKR1C3, CYP3A4, CYP3A43, HSD11B1, SULTIE1 and UGT1A8.

NGS technology allows for the detection of mutations and copy number variants. Although the hybrid capture method was used, we failed to assess copy number alterations because we sequenced only tumor samples. Sequencing coverage is uneven across the genome owing to variability introduced by the hybridization-capture step, and the development of a robust algorithm is challenging (34). On the other hand, multiplex PCR-based method fail to detection copy number changes and/or gene fusions (13). 
In summary, we performed WES of endometrial cancer samples and identified several potential cancer driver and passenger cancer genes (MAPT, IL24, MCM6, TSC1, BIRC2, CIITA, DST, CASP8, NOTCH2, ARMCX4, IGSF10, VPSI3C, DCT, DNAH14, TLN1, ZNF605, ZSCAN29, MOCOS, CMYA5, PCDH17, UGT1A8, CYFIP2, MACF1, NUDT5, JAKMIP1, PCDHGB4, FAM178A, SNX6, IMP4 and PCMTD1). The major limitation of this study was the small sample size.

\section{Acknowledgements}

The authors would like to thank Wei-Chi Wang (Health GeneTech Corporation, Taoyuan, Taiwan) for his invaluable assistance in bioinformatics analysis. The present study was supported by the National Health Research Institutes (NHRIEX104-10326BI), the Taiwan Ministry of Health and Welfare Clinical Trial Center (MOHW106-TDU-B-212-113004) and the China Medical University Hospital (DMR-106-105).

\section{References}

1. Sénéchal C, Cottereau E, de Pauw A, Elan C, Dagousset I, Fourchotte V, Gauthier-Villars M, Lae M, Stoppa-Lyonnet D and Buecher B: Environmental and genetic risk factors for endometrial carcinoma. Bull Cancer 102: 256-269, 2015 (In French).

2. Ashton KA, Proietto A, Otton G, Symonds I, McEvoy M, Attia J, Gilbert M, Hamann U and Scott RJ: Polymorphisms in genes of the steroid hormone biosynthesis and metabolism pathways and endometrial cancer risk. Cancer Epidemiol 34: 328-337, 2010.

3. Xu J, Lin X, Zhu H, Zhang Z and Yang B: Genetic variation of the CYP17 and susceptibility to endometrial cancer: A metaanalysis. Mol Biol Rep 40: 5085-5091, 2013.

4. Collaborative Group on Epidemiological Studies on Endometrial Cancer: Endometrial cancer and oral contraceptives: An individual participant meta-analysis of 27276 women with endometrial cancer from 36 epidemiological studies. Lancet Oncol 16: 1061-1070, 2015.

5. Trabert B, Wentzensen N, Yang HP, Sherman ME,Hollenbeck AR, Park Y and Brinton LA: Is estrogen plus progestin menopausal hormone therapy safe with respect to endometrial cancer risk? Int J Cancer 132: 417-426, 2013.

6. Brinton LA, Westhoff CL, Scoccia B, Lamb EJ, Trabert B Niwa S and Moghissi KS: Fertility drugs and endometrial cancer risk: Results from an extended follow-up of a large infertility cohort. Hum Reprod 28: 2813-2821, 2013.

7. Ong M,Carreira S, Goodall J, Mateo J, Figueiredo I, Rodrigues DN, Perkins G, Seed G, Yap TA, Attard G, et al: Validation and utilisation of high-coverage next-generation sequencing to deliver the pharmacological audit trail. Br J Cancer 111: 828-836, 2014.

8. André F, Bachelot T, Commo F, Campone M, Arnedos M, Dieras V, Lacroix-Triki M, Lacroix L, Cohen P, Gentien D, et al: Comparative genomic hybridisation array and DNA sequencing to direct treatment of metastatic breast cancer: A multicentre, prospective trial (SAFIR01/UNICANCER). Lancet Oncol 15 267-274, 2014

9. Cancer Genome Atlas Research Network: Comprehensive genomic characterization defines human glioblastoma genes and core pathways. Nature 455: 1061-1068, 2008

10. Bell D, Berchuck A, Birrer M, Chien J, Cramer DW, Dao F, Dhir R, DiSaia P, Gabra H, Glenn P, et al; Cancer Genome Atlas Research Network: Integrated genomic analyses of ovarian carcinoma. Nature 474: 609-615, 2011.

11. Cancer Genome Atlas Network: Comprehensive molecular characterization of human colon and rectal cancer. Nature 487: 330-337, 2012.

12. Kandoth C, Schultz N, Cherniack AD, Akbani R, Liu Y, Shen H, Robertson AG, Pashtan I, Shen R, Benz CC, et al; Cancer Genome Atlas Research Network: Integrated genomic characterization of endometrial carcinoma. Nature 497: 67-73, 2013.

13. Beadling C, Neff TL, Heinrich MC, Rhodes K, Thornton M, Leamon J, Andersen M and Corless CL: Combining highly multiplexed PCR with semiconductor-based sequencing for rapid cancer genotyping. J Mol Diagn 15: 171-176, 2013.
14. Grasso C, Butler T, Rhodes K, Quist M, Neff TL, Moore S, Tomlins SA, Reinig E, Beadling C, Andersen M, et al: Assessing copy number alterations in targeted, amplicon-based nextgeneration sequencing data. J Mol Diagn 17: 53-63, 2015.

15. Zheng Z, Liebers M, Zhelyazkova B, Cao Y, Panditi D, Lynch KD, Chen J, Robinson HE, Shim HS, Chmielecki J, et al: Anchored multiplex PCR for targeted next-generation sequencing. Nat Med 20: 1479-1484, 2014

16. Samorodnitsky E, Datta J, Jewell BM, Hagopian R, Miya J, Wing MR, Damodaran S, Lippus JM, Reeser JW, Bhatt D, et al: Comparison of custom capture for targeted next-generation DNA sequencing. J Mol Diagn 17: 64-75, 2015.

17. Drmanac R, Peters BA, Church GM, Reid CA and Xu X: Accurate whole genome sequencing as the ultimate genetic test. Clin Chem 61: 305-306, 2015.

18. Park JY, Clark P, Londin E, Sponziello M, Kricka LJ and Fortina P: Clinical exome performance for reporting secondary genetic findings. Clin Chem 61: 213-220, 2015.

19. Liang H, Cheung LW, Li J, Ju Z, Yu S, Stemke-Hale K, Dogruluk T, Lu Y, Liu X, Gu C, et al: Whole-exome sequencing combined with functional genomics reveals novel candidate driver cancer genes in endometrial cancer. Genome Res 22: 2120-2129, 2012.

20. Langmead B, Trapnell C, Pop M and Salzberg SL: Ultrafast and memory-efficient alignment of short DNA sequences to the human genome. Genome Biol 10: R25, 2009.

21. McKenna A, Hanna M, Banks E, Sivachenko A, Cibulskis K, Kernytsky A, Garimella K, Altshuler D, Gabriel S, Daly M, et al: The Genome Analysis Toolkit: A MapReduce framework for analyzing next-generation DNA sequencing data. Genome Res 20: 1297-1303, 2010

22. Sherry ST, Ward MH, Kholodov M, Baker J, Phan L, Smigielski EM and Sirotkin K: dbSNP: The NCBI database of genetic variation. Nucleic Acids Res 29: 308-311, 2001.

23. Landrum MJ, Lee JM, Riley GR, Jang W, Rubinstein WS, Church DM and Maglott DR: ClinVar: Public archive of relationships among sequence variation and human phenotype. Nucleic Acids Res 42 (D1): D980-D985, 2014.

24. Forbes SA, Beare D, Gunasekaran P, Leung K, Bindal N, Boutselakis H, Ding M, Bamford S, Cole C, Ward S, et al: COSMIC: Exploring the world's knowledge of somatic mutations in human cancer. Nucleic Acids Res 43 (D1): D805-D811, 2015.

25. Gao J, Aksoy BA, Dogrusoz U, Dresdner G, Gross B, Sumer SO, Sun Y, Jacobsen A, Sinha R, Larsson E, et al: Integrative analysis of complex cancer genomics and clinical profiles using the cBioPortal. Sci Signal 6: pl1, 2013.

26. Adzhubei IA, Schmidt S, Peshkin L, Ramensky VE, Gerasimova A, Bork P, Kondrashov AS and Sunyaev SR: A method and server for predicting damaging missense mutations. Nat Methods 7: 248-249, 2010

27. Kumar P, Henikoff S and Ng PC: Predicting the effects of coding non-synonymous variants on protein function using the SIFT algorithm. Nat Protoc 4: 1073-1081, 2009.

28. Hua X, Xu H, Yang Y, Zhu J, Liu P and Lu Y: DrGaP: A powerful tool for identifying driver genes and pathways in cancer sequencing studies. Am J Hum Genet 93: 439-451, 2013.

29. Chang YS, Huang HD, Yeh KT and Chang JG: Genetic alterations in endometrial cancer by targeted next-generation sequencing. Exp Mol Pathol 100: 8-12, 2016.

30. Hovelson DH, McDaniel AS, Cani AK, Johnson B, Rhodes K, Williams PD, Bandla S, Bien G, Choppa P, Hyland F, et al: Development and validation of a scalable next-generation sequencing system for assessing relevant somatic variants in solid tumors. Neoplasia 17: 385-399, 2015

31. Kwiatkowski DJ, Choueiri TK, Fay AP, Rini BI, Thorner AR de Velasco G, Tyburczy ME, Hamieh L, Albiges L, Agarwal N, et al: Mutations in TSC1, TSC2, and MTOR are associated with response to rapalogs in patients with metastatic renal cell carcinoma. Clin Cancer Res 22: 2445-2452, 2016.

32. Choschzick M, Tabibzada AM, Gieseking F, Woelber L, Jaenicke F, Sauter G and Simon R: BIRC2 amplification in squamous cell carcinomas of the uterine cervix. Virchows Arch 461: 123-128, 2012.

33. Ma XY, Ma CX and Wang JH: Endometrial carcinogenesis and molecular signaling pathways. Am J Mol Biol 4: 134-149, 2014.

34. Nam JY, Kim NK, Kim SC, Joung JG, Xi R, Lee S, Park PJ and Park WY: Evaluation of somatic copy number estimation tools for whole-exome sequencing data. Brief Bioinform 17: 185-192, 2016. 